\title{
MEASUREMENTS OF INTRA-BEAM SCATTERING GROWTH TIMES WITH GOLD BEAM BELOW TRANSITION IN RHIC**
}

\author{
W. Fischer, M. Bai, M. Blaskiewicz, J.M. Brennan, P. Cameron, R. Connolly, A. Lehrach, G. Parzen, \\ S. Tepikian, K. Zeno, and J. van Zeijts, Brookhaven National Lab, Upton, NY 11973, USA
}

\section{Abstract}

While RHIC is filled with beam, bunches are stored for up to several minutes at the injection energy before acceleration starts. In gold operation, the RHIC injection energy is below transition. A bunch length increase, and correspondingly an increase in the longitudinal emittance, can lead to particle loss during transition crossing and rebucketing into the storage buckets. The longitudinal growth of gold beams in RHIC at injection is dominated by intra-beam scattering. Measurements of longitudinal growth times are presented and compared with computations.

\section{INTRODUCTION}

The theory of intra-beam scattering was developed by Piwinsky [1], Bjorken and Mtingwa [2], Martini [3], Parzen [4], Wei [5] and others (see Ref. [6] for a more complete list). A number of computer codes were developed and tested with beam measurements [7, 8, 9]. With heavy ion beams at energies not attained thus far, the Relativistic Heavy Ion Collider (RHIC) allows measurements of intra-beam scattering in a new parameter regime.

Although intra-beam scattering affects all beam dimensions, we concentrate on the longitudinal growth time because the effect is most pronounced in this plane $[10,11]$. Furthermore, our transverse beam size measurement in not yet fully reliable and time-dependent emittance measurements were difficult to obtain.

In Ref. [5] growth times from intra-beam scattering are computed for the case of FODO cells only, which is a good approximation for RHIC. For bunched beam the longitudinal growth time $\tau_{s}$ is given by

$$
\tau_{s}^{-1}=\frac{1}{\sigma_{s}} \frac{d \sigma_{s}}{d t}=\frac{Z^{4} N_{b}}{A^{2}} \frac{r_{0}^{2} L_{c}(\beta c)}{8 \pi(\beta \gamma) \epsilon_{x} \epsilon_{y} \epsilon_{s}} F(\chi)\left(1-d^{2}\right),
$$

where $\sigma_{s}$ denotes the longitudinal rms length, $t$ time, $Z$ and $A$ charge and mass number of the ions respectively ( $Z=79$ and $A=197$ for gold), and $N_{b}$ the number of particles in the bunch. $r_{0}$ is the classical particle radius, $L_{c}$ is a form factor (which is approximately 20), $\beta$ and $\gamma$ the relativistic factors, and $c$ the speed of light. $\epsilon_{x}, \epsilon_{y}$ and $\epsilon_{s}$ are the normalized transverse and longitudinal rms emittances. If vertical dispersion can be neglected, one has

$$
d=\frac{D_{x} \sigma_{p}}{\left(\sigma_{x}^{2}+D_{x}^{2} \sigma_{p}^{2}\right)^{1 / 2}}, a=\frac{\beta_{x} d}{D_{x} \gamma}, b=\frac{\beta_{y} \sigma_{x}}{\beta_{x} \sigma_{y}} a, \chi=\frac{a^{2}+b^{2}}{2},
$$

where $D_{x}$ is the horizontal dispersion, $\sigma_{x, y, p}$ the transverse and momentum rms beam sizes respectively, and $\beta_{x, y}$ the

${ }^{*}$ Work performed under the auspices of the US department of Energy. transverse $\beta$-functions. The function $F(\chi)$ is defined by

$$
F(\chi)=\frac{(1+2 \chi) I(\chi)-3}{1-\chi}
$$

with

$$
I(\chi)= \begin{cases}\frac{1}{\sqrt{\chi(\chi-1)}} \operatorname{arctanh} \sqrt{\frac{\chi-1}{\chi}} & \chi \geq 1 \\ \frac{1}{\sqrt{\chi(1-\chi)}} \arctan \sqrt{\frac{1-\chi}{\chi}} & \chi<1\end{cases}
$$

During the run 2000, gold ions were injected with energies corresponding to $\gamma=10.25$. Measurements of freely expanding single bunches were carried out in the Blue ring, and extended over up to half an hour. From 12 measured cases, a bunch length increase was observed in 5 cases. In the other cases a longitudinal misinjection lead to an initially filled bucket for which the rms bunch length decreases. We concentrate on the cases that exhibit a growing bunch length since these can be compared with results from existing intra-beam scattering codes.

\section{MEASUREMENTS}

Longitudinal and vertical beam sizes were observed over up to half an hour as the beam expanded. The longitudinal size was measured with a wall current monitor (WCM) and the transverse size with an ionization profile monitor (IPM).

Longitudinal Beam Size The wall current monitors give the instantaneous beam current as a function of time. The resolution of our devices is $0.25 \mathrm{~ns}$. Wall current monitors were used for two purposes. First, the bunched beam intensity was measured, and second, the longitudinal bunch

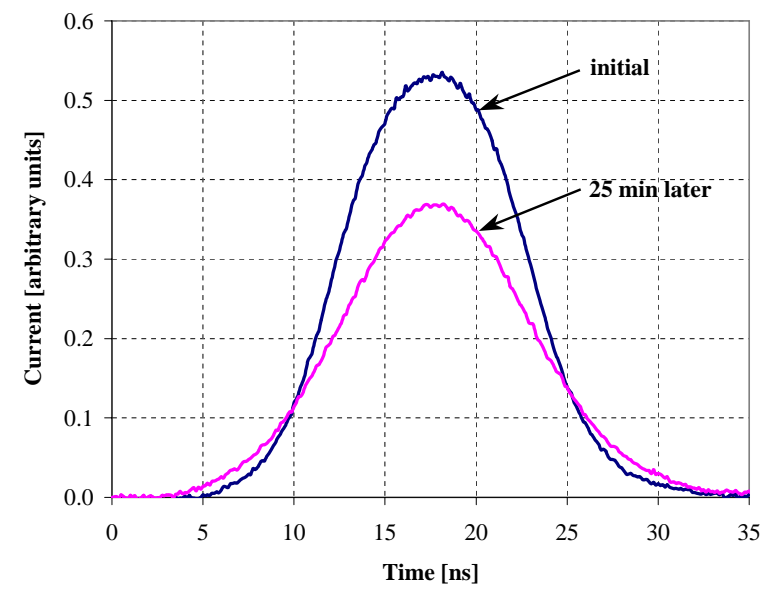

Figure 1: Longitudinal profile at the beginning and at the end of a measurement. The horizontal scale corresponds to one bucket length. 
length was determined as a function of time. The longitudinal profiles were fitted with a Gaussian function to obtain the rms length.

Longitudinal profiles at the beginning and end of a measurement are shown in Fig. 1. In addition to the reduction in beam intensity, the growth in bunch length is visible.

Transverse Beam Size Beam profiles were measured with the horizontal and vertical ionization beam profile monitor (IPM) in the Blue ring. An IPM measures the distribution of electrons in the beam line resulting from residual gas ionization during bunch passage. The electrons are swept transversely from the beam line and collected on 64 strip anodes oriented parallel to the beam axis. At each bunch passage the charge pulses are amplified, integrated, and digitized. The profiles used here were produced by averaging 200 consecutive turns.

The IPMs performed well during the 1999 commissioning run and early in the 2000 run. However as the bunch intensity increased there was a beam-induced ringing that increased in amplitude until it saturated the amplifiers and made the IPM's unusable. This ringing was traced to a copper-mesh window placed over the micro channel plate input and capacitively grounded around its perimeter to prevent beam-anode coupling. When a beam bunch passed it induced a low-frequency longitudinal oscillation in the rf screen which was picked up by the anodes. Near the end of the run the vertical IPM in the Blue ring was fixed in time for the measurements reported here. Fig. 2 shows the time evolution of the measured vertical rms beam sizes. During the first $200 \mathrm{~s}$ of case 8 and case 9 the charge sensitive amplifiers for center plate channels were saturated and the beam size is overstated. After amplification adjustment the correct beam size is shown. Due to the ringing problem the horizontal data are not reliable.

For the simulations we use the average of all vertical emittance measurements for both the horizontal and vertical emittance since the machine was fully coupled in all cases. The average normalized vertical emittance was $13 \mu \mathrm{m}(95 \%)$.

\section{COMPUTATIONS OF INTRA-BEAM SCATTERING GROWTH TIMES}

We use two programs to compare measured longitudinal intra-beam scattering growth times with computations. Program 1 is written by G. Parzen and is based on Refs. [3, 4]. Program 2 is written by J. Wei and is based on Ref. [5].

Simulations Program 1 In Ref. [3] it is assumed that the initial distribution of the particles in the transverse and longitudinal coordinates are Gaussian and remain Gaussian, while $\epsilon_{x}, \epsilon_{y}, \sigma_{p}$ change with time as a result of intrabeam scattering. One can then find expressions for the growth rates $d \epsilon_{x} / d t, d \epsilon_{y} / d t, d \sigma_{p} / d t$. The expressions for the growth rates contain a multiple integral which has to be evaluated numerically for each element of the lattice and

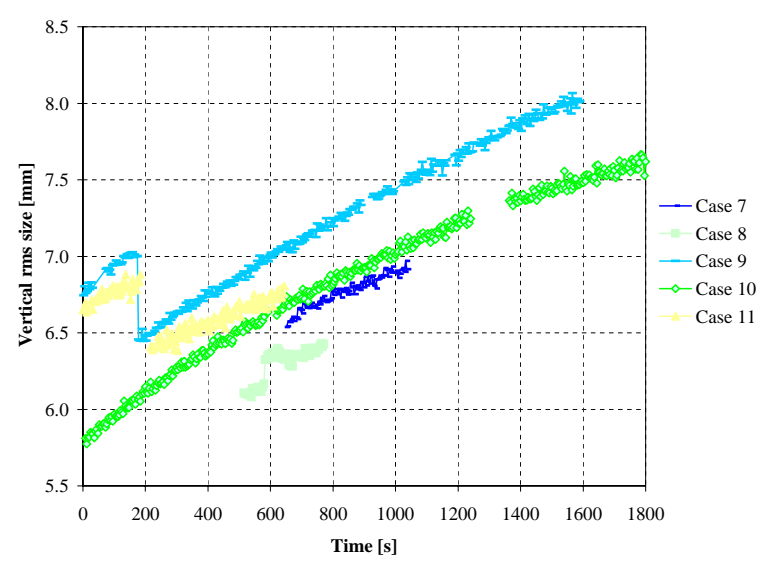

Figure 2: Vertical beam size growth as observed with the ionization profile monitor. Shown are the standard deviations of fitted Gaussian distributions as a function of time. During the first $200 \mathrm{~s}$ of case 8 and case 9 the beam size is overstated due to amplifier saturation.

averaged over the accelerator. This is done using the lattice of the accelerator. The expressions for the growth rates can be integrated in time to find $\epsilon_{x}, \epsilon_{y}, \sigma_{p}$ at any later time. The results of the theory have been tested experimentally $[7,8]$.

Simulations Program 2 In the code it is assumed that the whole machine consists of FODO cells. The input consists of the FODO cell parameters, particle parameters like mass and charge state, beam parameters such as transverse and longitudinal emittances and the bunched intensity. The program calculates the longitudinal and transverse beam dimensions as a function of time along with the longitudinal and transverse growth times.

Results Only five of the twelve measured cases show a longitudinally growing bunch. To measure the growth time in Eq. (1), the rms bunch length curves were fitted with a polynomial from which the growth times could be determined analytically. The fitted curves are shown in Fig. 3 and the obtained growth times are shown in Tab. 1. Typically a polynomial of fifth order was used for the fit. An estimate for the error of the measured growth rates can be obtained by fitting polynomials of different orders to the experimental data. When polynomials of order two to five are used, the obtained growth rates vary by up to $35 \%$ around the average value, while the $R^{2}$ of the fit changes only little. Note that growth rates and growth times are derivatives of a measured function and therefore sensitive to small local changes in the measured function.

For all cases for which a bunch length growth was observed, the bunch length growth was also computed with both programs using the initial bunch length, transverse sizes, and intensity. For both the horizontal and vertical emittance, the average measured vertical emittance was used in the computations. The machine was fully coupled in all cases and the vertical emittance measurement was much more reliable than the horizontal one. The growth time was computed with the beam parameters at the begin- 
Table 1: Comparison of measured and computed longitudinal growth times $\tau_{s}$ from intra-beam scattering.

\begin{tabular}{|c|c|c|c|c|c|c|c|c|c|c|}
\hline \multirow[t]{3}{*}{ Case } & \multicolumn{5}{|c|}{ initial } & \multicolumn{5}{|c|}{ final } \\
\hline & \multirow{2}{*}{$\begin{array}{c}\text { measured } \\
\tau_{s, m} \\
{[\mathrm{~min}]}\end{array}$} & \multicolumn{2}{|c|}{ simulation 1} & \multicolumn{2}{|c|}{ simulation 2} & \multirow{2}{*}{$\begin{array}{c}\text { measured } \\
\tau_{s, m} \\
{[\mathrm{~min}]}\end{array}$} & \multicolumn{2}{|c|}{ simulation 1} & \multicolumn{2}{|c|}{ simulation 2} \\
\hline & & $\begin{array}{c}\tau_{s, c} \\
{[\mathrm{~min}]}\end{array}$ & $\begin{array}{c}\frac{\tau_{s, c}}{\tau_{s, m}}-1 \\
{[\%]}\end{array}$ & $\begin{array}{c}\tau_{s, c} \\
{[\mathrm{~min}]}\end{array}$ & $\begin{array}{c}\frac{\tau_{s, c}}{\tau_{s, m}}-1 \\
{[\%]}\end{array}$ & & $\begin{array}{c}\tau_{s, c} \\
{[\mathrm{~min}]}\end{array}$ & $\begin{array}{c}\frac{\tau_{s, c}}{\tau_{s, m}}-1 \\
{[\%]}\end{array}$ & $\begin{array}{c}\tau_{s, c} \\
{[\mathrm{~min}]}\end{array}$ & $\begin{array}{c}\frac{\tau_{s, c}}{\tau_{s, m}}-1 \\
{[\%]}\end{array}$ \\
\hline 1 & 53 & 114 & 115 & 120 & 125 & 179 & 199 & 11 & 332 & 85 \\
\hline 2 & 183 & 335 & 94 & 366 & 100 & 361 & 426 & 18 & 493 & 37 \\
\hline 3 & 208 & 316 & 52 & 357 & 73 & 273 & 405 & 48 & 430 & 58 \\
\hline 11 & 110 & 127 & 15 & 136 & 23 & 464 & 290 & -37 & 357 & -23 \\
\hline 12 & 165 & 167 & 1 & 171 & 4 & 361 & 324 & -10 & 370 & 3 \\
\hline
\end{tabular}

ning and end of a measurement. The computed longitudinal growth times are shown in Tab. 1.

In all but one instance (case 11, final), the computed growth time is larger than the measured growth time. Computed growth times exceed measured growth times by up to $125 \%$, with an average of $50 \%$. This constitutes a satisfactory agreement given the $35 \%$ error in the growth time measurement.

In Fig. 3 the bunch length development of the five simulated cases is shown. The measured and computed bunch lengths are depicted. Except for the case starting with the smallest bunch length (case 1) there is a relatively good agreement. Case 1 is the case with the largest growth rate and a source other than intra-beam scattering may have contributed significantly to the bunch length increase. Note that in the simulations the particle loss observed in the measurements is disregarded, which artificially increases the computed bunch length growth rate by up to $30 \%$ at the end of the computation period. This error has the same magnitude as the measurement error for the growth rates.

The results of simulation program 1 and simulation program 2 show one significant difference in the transverse growth rate. Program 1 predicts a slowly increasing beam size in both transverse planes while program 2 predicts a beam that is shrinking in both transverse dimensions. A transversely shrinking beam is expected if the bunch behaves like a gas in which energy is transfered from the hotter transverse dimensions to the cooler longitudinal dimension. However, no transverse shrinkage was observed, the beam grew transversely.

\section{SUMMARY}

Emittance growth of bunched gold beams were observed at injection. Observed growth rates vary significantly from measurement to measurement. The most significant mechanism for longitudinal bunched beam growth is intra-beam scattering. Comparisons with two computer codes give an agreement between measured and computed growth times within a factor of two or better.

\section{ACKNOWLEDGMENTS}

The authors are thankful to the operations, instrumentation, radio frequency, control and accelerator physics teams that supported the data acquisition, storage, processing and analysis in many ways. In addition, we are thankful to J. Wei and $\mathrm{H}$. Hahn for discussions.

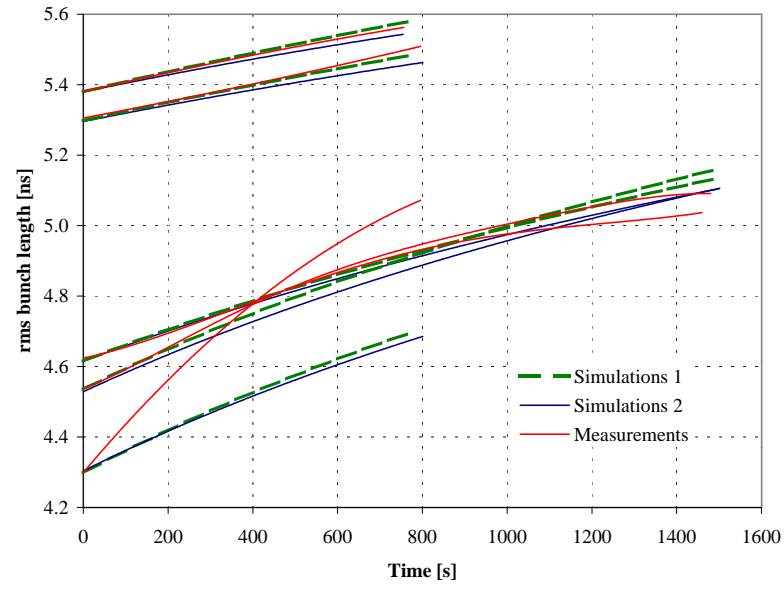

Figure 3: Comparison between measured and computed bunch length growth.

\section{REFERENCES}

[1] A.Piwinski, HEAC 74, Stanford, pp. 405 (1974).

[2] J.D. Bjorken and S.K. Mtingwa, "Intrabeam Scattering", Part. Accel. Vol. 13, pp. 115-143 (1983).

[3] M. Martini, "Intrabeam Scattering in the ACOL-AA Machines", CERN PS/84-9 (AA) (1984).

[4] G. Parzen, "Intrabeam Scattering at High Energies", Nuc. Inst. Meth. A245, pp. 231-240 (1986).

[5] J. Wei, "Evolution of Hadron Beams under Intrabeam Scattering", PAC 93, Washington, D.C. pp. 3561 (1993).

[6] A. Piwinski, "Touschek Effect and Intrabeam Scattering" in "Handbook of Accelerator Physics and Engineering" edited by A.W. Chao and M. Tigner, World Scientific (1999).

[7] M. Conte and M. Martini, "Intrabeam Scattering in the CERN Anitproton Accumulator", Part. Accel. 17, pp. 1-10 (1985).

[8] L.R. Evans and J. Gareyte, PAC 85 (1985).

[9] C. Bhat, L.K. Spentzouris and P.L. Colestock, "Measurements of Intrabeam Scatterign Rates below Transition in the Fermilab Antiproton Accumulator", PAC 99, New York (1999).

[10] J. Wei, "Intra Beam Scattering in RHIC", BNL RHIC/AP/63 (1995).

[11] W. Fischer et al. "Emittance Growth in RHIC During Injection", proceedings of the LHC96 Workshop on High Brightness Beams for Large Hadron Colliders, Part. Acc. Vol. 58, pp. 181-191 (1997).

[12] W. Fischer, M. Bai, J.M. Brennan, M. Blaskiewicz, P. Cameron, R. Connolly, A. Lehrach, G. Parzen, S. Tepikian, J. v. Zeijts and K. Zeno, "Beam Growth Measurements in RHIC with Gold at Injection", BNL C-A/AP/44 (2001). 